\title{
Lack of HER-2 gene amplification and association with pathological and clinical characteristics of differentiated thyroid cancer
}

\author{
WAHID MDAH ${ }^{1}$, RANEEN MZALBAT $^{2}$, PETER GILBEY $^{1}$, MOSHE STEIN $^{3}$, \\ ADI SHARABI ${ }^{2}$ and JAMAL ZIDAN ${ }^{2,4}$ \\ ${ }^{1}$ Otolaryngology, Head and Neck Surgery Unit, ${ }^{2}$ Oncology Institute, Ziv Medical Center, Safed; ${ }^{3}$ Department of Oncology, \\ Rambam Medical Center, ${ }^{4}$ The Faculty of Medicine, Galilee, Bar-Ilan University, Haifa, Israel
}

Received September 19, 2012; Accepted July 3, 2014

DOI: $10.3892 / \mathrm{mco} .2014 .346$

\begin{abstract}
Human epidermal growth factor receptor 2 (HER-2) is a well recognized prognostic and predictive factor in breast cancer. However, the role of HER-2 in thyroid cancer remains controversial. The aim of this study was to evaluate HER-2 expression in differentiated thyroid cancer (DTC) and determine whether there is an association with other clinical and pathological characteristics. A total of 69 patients with DTC were investigated, 58 of whom had papillary and 11 follicular carcinomas. HER-2 was detected by immunohistochemical examination on sections from formalin-fixed, paraffin-embedded tumor tissues. Tumors with HER-2 expression classed as +1 and +2 were retested with chromogenic in situ hybridization. Clinicopathological data were retrieved from the hospital records of the patients. HER-2 overexpression was found in $4(6.9 \%)$ of the 58 patients with papillary carcinoma, whereas there was no HER-2 overexpression in any of the 11 cases of follicular carcinoma. There was no association of HER-2 expression with tumor size, pathological grade and cervical lymph node metastasis. In conclusion, there were no HER-2 positive cases of follicular carcinoma and the incidence of HER-2 overexpression in papillary carcinoma was very low. Thus, HER-2 cannot be used routinely as a prognostic or predictive factor in DTC. The expression of other epidermal growth factor receptors in DTC merits further investigation.
\end{abstract}

\section{Introduction}

Human epidermal growth factor receptor 2 (HER-2) is a 185-kDa transmembrane glycoprotein with tyrosine kinase activity. HER-2 is encoded by the c-erbB-2 proto-oncogene (1). HER-2 overexpression was first detected in human breast and

Correspondence to: Professor Jamal Zidan, Oncology Institute, Ziv Medical Center, POB 1008, Safed 13000, Israel

E-mail: zidan.j@ziv.health.gov.il

Key words: human epidermal growth factor receptor 2 expression, clinicopathological characteristics, differentiated thyroid cancer ovarian cancers (2) and was also demonstrated in a wide range of human adenocarcinomas originating in different organs, such as the stomach, colon, lung and pancreas (3-5). HER-2 is generally an important factor for the growth and progression of malignant tumors and its overexpression in lung and breast cancer appears to be associated with an increased metastatic potential of the cells (6). The greatest value of HER-2 status as a predictive marker is in the prediction of response to therapies that specifically target HER-2, such as trastuzumab and lapatinib, in breast and gastric cancer (7-9).

Differentiated thyroid cancer (DTC), including papillary and follicular subtypes, accounts for $90 \%$ of all thyroid malignancies (10). DTC is generally associated with good prognosis and long-term survival rates $>90 \%$ (11). However, despite their favorable prognosis, patients with DTC are at risk of tumor recurrence for several decades from the time of diagnosis. The therapeutic management of recurrent and metastatic DTC depends on the type of the initial treatment, the location and extent of the disease. Radioactive iodine $\left({ }^{131} \mathrm{I}\right)$ administration and/or surgical excision of the lesions remain the first and main approach (12). A number of advanced thyroid cancers eventually develop lack of iodine avidity. Until recently, chemotherapy was the only option for systemic treatment, but only achieved low response rates and some metastatic patients were referred for best supportive care.

A element common to thyroid cancers is their vascularity, with elevated levels of vascular endothelial growth factor compared to those in normal thyroid tissue, which is the rationale behind the use of antiangiogenic drugs, such as axitinib and sorafenib, in the treatment of advanced DTC. These two drugs were found to achieve response rates of 30 and $23 \%$ respectively $(13,14)$.

There is no consensus in the currently available literature regarding the expression of HER-2 and its prognostic and predictive value in thyroid cancer, although expression rates of $0-50 \%$ were reported by previous studies $(15,16)$. These contradictory findings prompted us to investigate the HER-2 expression status in a retrospective series of DTC, including follicular and papillary subtypes.

Our second aim was to determine the association between HER-2 expression and the clinicopathological characteristics 
of DTC and determine whether HER-2 may be used as a prognostic factor and/or as a target for treatment with biological-targeted therapy with trastuzumab or other drugs in DTC patients.

\section{Patients and methods}

Patients and tumors. In this retrospective study, biopsies were collected from surgically removed DTCs from 69 patients treated at the Ziv Medical Center between 1999 and 2007. The patients exhibited no distant (visceral) metastases at the time of diagnosis. Histological classification was performed at our hospital according to the World Health Organization classification (17). Patient data at all the stages of the disease were retrospectively collected and summarized. The hospital records provided clinical and demographic data that included ethnicity, histology, age, surgical procedure, physical findings and disease stage according to the International Union Against Cancer (18). The pathological data included primary tumor diameter, histological type, tumor size, number of lymph nodes involved and tumor grade. HER-2 expression was evaluated using immunohistochemistry (IHC) or chromogenic in situ hybridization (CISH) if HER-2 expression was classed as +1 and +2 in IHC. Formalin-fixed, paraffin-embedded (FFPE) tissue was used for both tests. HER-2 was considered to be overexpressed if it was classed as +3 by IHC, using the scoring system described below, or if positive amplification was shown in the CISH test.

IHC. IHC was performed using the HercepTest (DakoCytomation, Carpinteria, CA, USA), following the manufacturer's instructions. Briefly, this procedure includes deparaffinization and rehydration steps, followed by an epitope retrieval step, in which the tissue sample is incubated in EDTA buffer solution at $>95^{\circ} \mathrm{C}$ for $20 \mathrm{~min}$. The slide is then subjected to a series of alternating washes in phosphate-buffered saline (PBS) and incubation steps, beginning with a peroxidase-blocking reagent for $10 \mathrm{~min}$ and followed by a polyclonal antibody to HER-2 (A0485; DakoCytomation) applied to the sections at a 1:200 dilution in PBS and incubated for $60 \mathrm{~min}$ at room temperature, followed by a visualization reagent (dextran polymer) conjugated with horseradish peroxidase for $30 \mathrm{~min}$ and finally with a 3,3'-diaminobenzidine chromogen solution for $10 \mathrm{~min}$. After a final wash, the slide is counterstained with hematoxylin.

Tumor cells with circumferential membranous positivity were considered as overexpressing the HER-2 protein and scoring was performed according to the HercepTest manufacturer's recommendations by pathologists experienced in breast pathology and immunohistochemical interpretation of HER-2 testing. According to the HercepTest criteria, immunoreaction was scored as follows: $+3,>10 \%$ of tumor cells exhibiting strong and complete membranous staining; +2, moderate and complete membrane positivity in $>10 \%$ cells; +1 , weak and incomplete membrane positivity in $>10 \%$ cells; and 0 , membrane staining absent or present in $<10 \%$ cells. Tumors scored as +3 were considered to be HER-2-positive, whereas tumors scored as $0 /+1$ were designated as HER-2-negative. In +2 tumors, as determined by IHC, CISH analysis was performed.
Table I. Patients characteristics.

Characteristics

No. $(\%)$

Age at diagnosis (years)
Mean
Range

$44.6 \pm 14.4$

Gender

Female

Male

Histology

Papillary carcinoma

Follicular carcinoma

Type of operation

Total thyroidectomy

Subtotal thyroidectomy

Near-total thyroidectomy

Hemithyroidectomy

${ }^{131}$ I ablation after surgery

Yes

65 (94.2)

No

${ }^{131} \mathrm{I}$, radioactive iodine.

CISH. CISH allows detection of gene amplification, chromosome translocation and chromosome number using conventional peroxidase reactions under a bright-field microscope on FFPE tissues cut in 4-5-mm sections. The essence of CISH is the ability of labeled nucleic acid probes to hybridize, in situ, to specific sections of complementary nucleic acid in the sample. The probe hybridization results may be visualized within the context of the surrounding tissue morphology. Therefore, pathologists may view tissue morphology and gene aberrations simultaneously (18). The 84-0146 SPoT-Light1 HER-2 CISH $^{\text {TM }}$ kit (Zymed Laboratories, Invitrogen immunodetection, San Francisco, CA, USA) was used. The CISH staining results may be clearly visualized under a standard bright-field microscope and a 406 dry lens. Tumors with HER-2 gene amplification typically appear as a large peroxidase-positive intranuclear gene copy clusters, as numerous individual peroxidase-positive small signals, or as a mixture of clusters and individual gene copies. Tumors with a normal HER-2 gene status typically exhibit two signals or dots/nucleus.

Statistical analysis. The data were analyzed in association with histological type and other clinicopathological parameters. For evaluating clinical and pathological parameters, and the association with HER-2 levels, the $\chi^{2}$ test was used. $\mathrm{P}<0.05$ was considered to indicate a statistically significant difference.

\section{Results}

Patient characteristics. A total of 69 cases were investigated and the patient characteristics are presented in Table I. The mean age at diagnosis was 44.6 years and $76.8 \%$ of the patients were female. Papillary carcinoma was more common 
Table II. Stage of the disease at diagnosis.

\begin{tabular}{lrl}
\hline Characteristics & No. $(\%)$ \\
\hline Size of primary tumor & & \\
T1 & 44 & $(63.8)$ \\
T2 & 13 & $(18.8)$ \\
T3 & 10 & $(14.5)$ \\
T4 & 2 & $(2.9)$ \\
Lymph node status & & \\
N0 & 58 & $(84.0)$ \\
N1 & 11 & $(16.0)$ \\
Distant metastases & & \\
Lungs & 1 & $(1.5)$ \\
Capsular invasion & & \\
No & 50 & $(72.5)$ \\
Yes & 19 & $(27.5)$ \\
\hline
\end{tabular}

compared to follicular carcinoma (84.1 and $15.9 \%$, respectively). The majority of the patients were subjected to total or subtotal thyroidectomy. Of all the patients, $94.2 \%$ were treated with ${ }^{131}$ I ablation after surgery. In $63.8 \%$ of the patients the tumor was sized $<2 \mathrm{~cm}$ and $15.9 \%$ had lymph node metastases (Table II). One patient had lung metastases diagnosed 4 months after the operation.

HER-2 expression and correlation with clinicopathological characteristics. In $4(6.9 \%)$ of the 58 patients with papillary carcinoma, HER-2 was overexpressed $(+3)$ but the proportion of patients was not considered significant. One of these 4 patients was male and another patient had lymph node metastases. There was no HER-2 overexpression in any of the 11 patients with follicular carcinoma (Table III).

Five tumors were scored as +2 and 10 as +1 by IHC for HER-2. All these tumors were evaluated by CISH. No gene amplification was found in either of these tumors. There was no correlation between HER-2 expression and tumor size, lymph node metastases, age and gender.

\section{Discussion}

The role of the HER-2 proto-oncogene in thyroid cancer remains controversial, particularly regarding the prognostic significance of its amplification and overexpression. Haugen et al (16) investigated the c-erbB-2 protein expression in frozen thyroid cancer specimens and detected immunoreactivity at the cell membrane as well as the cytoplasm in papillary thyroid carcinomas. Additionally, in those specimens, the HER-2/neu mRNA levels were analyzed and found to be significantly elevated. Kremser et al (19) observed a highly significant correlation between the HER-2/neu expression in papillary and follicular thyroid carcinomas and the clinical course regarding metastatic disease. Both tumor entities without distant metastases exhibited non-significant cytoplasmic immunostaining compared to patients with metastases. There was no association identified between patient gender and HER-2 expression, although a significant
Table III. HER-2 expression in thyroid cancer tissue.

\begin{tabular}{lc}
\hline HER-2 status & No. $(\%)$ \\
\hline Papillary carcinoma $(\mathrm{n}=58)$ & $54(93.1)$ \\
Negative & $4(6.9)$ \\
Positive & \\
Follicular carcinoma $(\mathrm{n}=11)$ & $11(100.0)$ \\
Negative & $0(0.0)$ \\
Positive & \\
Total no. $(\mathrm{n}=69)$ & $65(94.2)$ \\
Negative & $4(5.8)$ \\
Positive & \\
\hline
\end{tabular}

correlation with age was identified. Advanced tumor stage presented with a tendency towards higher HER-2 expression. Cervical lymph node metastases did not correlate with HER-2 levels. Furthermore, there was no correlation between HER-2 expression and any site of metastasis, histological subtype or variant (19).

Similar to our findings, previous studies reported no HER-2 overexpression in thyroid tumors. Lemoine et al (15) investigated a large series of benign and malignant thyroid tumors by means of Southern blot analysis, polymerase chain reaction and $\mathrm{IHC}$, without detection of abnormalities in structure or expression of c-erbB-2, and concluded that activation of this oncogene is not necessarily involved in neoplastic transformation or the prognosis of thyroid tumors. However, in our cases, 4 of the $58(6.9 \%)$ patients with papillary carcinoma overexpressed HER-2, as determined by IHC. No correlation was found in these 4 cases between HER-2 and tumor size, lymph node metastasis, age and gender. In addition, there was no HER-2 overexpression in the 11 patients with follicular carcinoma.

Utrilla et al (20) investigated 67 thyroid tumors (10 follicular adenomas, 11 follicular carcinomas, 3 anaplastic carcinomas, 25 papillary carcinomas, 18 medullary carcinomas and 16 metastases) for HER-2 expression by IHC using an antigen aretrieval method for paraffin-embedded material and a specific polyclonal antibody against the intracytoplasmic part of the HER-2 oncoprotein. No expression of this oncoprotein was detected in follicular adenomas, follicular carcinomas and anaplastic carcinomas; however, $52 \%$ of the papillary carcinomas (some membranous staining, but mostly diffuse cytoplasmic staining patterns) and all the medullary carcinomas (granular cytoplasmic staining pattern) were immunopositive. Sugg et al (21) reported no DNA amplifications of the c-erbB-2 gene in 47 human thyroid tumors, including papillary and follicular carcinoma. There was also no membrane staining with NCL-CBE1, a monoclonal antibody specific for the external domain of HER-2. Consistent with previous studies, they found that the cytoplasmic immunoreactivity of HER-2 correlates with differentiation of DTCs and may be a prognostic factor in this disease. The occurrence of cytoplasmic immunoreactivity of HER-2 has not been fully elucidated. De Potter et al (22) discovered a 155-kDa (p155) 
protein reacting with the antibody. p155 may be localized in the mitochondrial cristae, explaining the partly granular aspect of the staining. In bladder cancer, c-erbB-2 amplification, high mRNA levels and strong cytoplasmic immunoreactivity were observed, correlating with the high levels of p155. This may be attributed to reactions with a homologue of the c-erbB-2 protein, which may be due to an alteration or instability of the oncoprotein itself or the mRNA (23).

In conclusion, according to the present study and other available publications, HER-2 is not considered suitable to be used routinely as a prognostic or predictive factor in DTC. We detected HER-2 overexpression in only a few cases of papillary thyroid carcinoma, but its levels do not appear to correlate with other parameters of aggressive behavior. There were no HER-2-positive cases of follicular carcinoma. Therefore, only a limited number of patients with papillary DTC refractory to ${ }^{131}$ I may have HER-2-positive tumors and may benefit from biological-targeted therapy. We are planning a new study to evaluate HER-2 expression in metastatic DTC. However, the profile of other epidermal growth factor receptors in different thyroid cancer types requires further investigation.

\section{References}

1. Allgayer H, Babic R, Gruetzner KU, Tarabichi A, Schildberg FW and Heiss MM: C-erbB-2 is of independent prognostic relevance in gastric cancer and is associated with the expression of tumor-associated protease systems. J Clin Oncol 18: 2201-2209, 2000.

2. Zhang X, Silva E, Gershenson D and Hung MC: Amplification and rearrangement of c-erb B proto-oncogenes in cancer of human female genital tract. Oncogene 4: 985-989, 1989.

3. Yokota J, Yamamoto T, Toyoshima K, et al: Amplification of c-erbB-2 oncogene in human adenocarcinomas in vivo. Lancet 8484: 765-767, 1986.

4. Hall PA, Hughcs CM, Staddon SL, Richman PI, Gullick WJ and Lemoine NR: The c-erbB-2 proto-oncogene in pancreatic cancer J Pathol 161: 195-200, 1990.

5. D'Emilia J, Bulovas K, D'Ercole K, Wolf B, Steele G Jr and Summerhayes IC: Expression of the c-erbB-2 gene product (185) at different stages of neoplastic progression in the colon. Oncogene 4: 1233-1239, 1989.

6. Tan M, Yao J and Yu D: Overexpression of the c-erbB-2 gene enhanced intrinsic metastasis potential in human breast cancer cells without increasing their transformation abilities. Cancer Res 57: 1199-1205, 1997.
7. Slamon DJ, Clark GM, Wong SG, Levin WJ, Ullrich A and McGuire WL: Human breast cancer: correlation of relapse and survival with amplification of the HER-2/neu oncogene. Science 235: 177-182, 1987.

8. Geyer C, Forster, Lindquist D, et al: Lapatinib plus capecitabine for HER2-positive advanced breast cancer. N Engl J Med 355: 2733-2743, 2006.

9. Bang YJ, Van Cutsem E, Feyereislova A, et al: Trastuzumab in combination with chemotherapy versus chemotherapy alone for HER2-positive advanced gastric or gastro-esophageal junction cancer (ToGA): a phase 3, open-label, randomized controlled trial. Lancet 376: 687-697, 2010.

10. Green LD, Mack L and Pasieka JL: Anaplastic thyroid cancer and primary thyroid lymphoma: A review of these rare thyroid malignancies. J Surg Oncol 94: 725-736, 2006.

11. Gilliland FD, Hunt WC, Morris DM and Key CR: Prognostic factors for thyroid carcinoma: a population-based study of 15,698 cases from the Surveillance, Epidemiology and End Results (SEER) program 1973-1991. Cancer 79: 564-573, 1997.

12. Sherman SI: Thyroid carcinoma. Lancet 361: 501-5011, 2003.

13. Cohen E, Rosen L, Vokes EE, et al: Axitinib is an active treatment for all histologic subtypes of advanced thyroid cancer: Results from a phase II study. J Clin Oncol 26: 4708-4713, 2008.

14. Gupta-Abramson V, Troxel A, Nellore A, et al: Phase II trial of sorafenib in advanced thyroid cancer. J Clin Oncol 26: 4714-4719, 2008.

15. Lemoine NK, Wyllie FS, Lillehaug JR, et al: Absence of abnormalities of the c-erbB-1 and c-erbB-2 proto-oncogenes in human thyroid neoplasia. Eur J Cancer 26: 777-779, 1990.

16. Haugen DR, Akslen LA, Varhaug JE and Lillehaug JR: Expression of c-erbB-2 protein in papillary thyroid carcinomas. Br J Cancer 65: 832-837, 1992.

17. Hedinger C, Williams ED and Sobin LH: The WHO histological classification of thyroid tumors: a commentary on the second edition. Cancer 63: 908-911, 1989.

18. UICC: International Union Against Cancer: TNM Classification of Malignant Tumours. 6th edition. John Wiley \& Sons, Inc., New York, pp131-141, 2002.

19. Kremser R, Obrist P, Spizzo G, et al: Her2/neu overexpression in differentiated thyroid carcinomas predicts metastatic disease. Virchows Arch 442: 322-328, 2003.

20. Utrilla JC,Martin-Lacave I,San Martin MV,Fernandez-Santos JM and Galera-Davidson $\mathrm{H}$ : Expression of c-erbB-2 oncoprotein in human thyroid tumours. Histopathology 34: 60-65, 1999.

21. Sugg SL, Ezzat S, Zheng L, Rosen IB, Freeman JL and Asa SL: Cytoplasmic staining of erbB-2 but not mRNA levels correlates with differentiation in human thyroid neoplasia. Clin Endocrinol 49: 629-637, 1998.

22. de Potter CR, Van Daele S, Van de Vijver MJ, et al: The expression of the neu oncogene product in breast lesions and in normal fetal and adult human tissues. Histopathology 15: 351-362, 1989.

23. Coombs LM, Pigott DA, Sweeney E, et al: Amplification and overexpression of c-erbB-2 in transitional cell carcinoma of the urinary bladder. Br J Cancer 63: 601-608, 1991. 